\title{
A BASELINE STUDY OF A LOW-COST, HIGH-RESOLUTION, IMAGING SYSTEM USING WAVEFRONT RECONSTRUCTION
}

\author{
Shawn E. Gano * Aero 483/583 Winter 2001 Class $^{\dagger} \quad$ David C. Hyland ${ }^{*} \quad$ Pierre T. Kabamba ${ }^{\S}$ \\ Department of Aerospace Engineering \\ University of Michigan \\ Ann Arbor, Michigan
}

\begin{abstract}
$\underline{\text { Abstract }}$
Current high-resolution imaging systems used in space applications are very large in size and mass. These systems tend to have limited resolution because the mirrors can only be manufactured up to certain sizes due to surface accuracy requirements. Furthermore, the masses of such mirrors tend to grow as the fourth power of their diameters, which is directly linked to the total system cost. The imaging system presented in this paper removes the size of the mirrors as the design driver. This is done by using a sparse aperture optical bench to fill a Modulation Transfer Function equivalent to that of a single aperture with seven times the diameter of each of its mirrors. Also an emerging technology, the so called "Silicon Eye", is used to reconstruct the light wavefront and image, and allows for the possibility of holograms.
\end{abstract}

*Paper Contact: sgano@engin.umich.edu (before July 1, 2001), sgano@nd.edu (after July 1, 2001), Student Member AIAA

$\dagger$ Joong-Huan Bahng, Kevin Bernas, Gail Booren, Sarah Brittain, Stephen Broschart, Cameron Chappell, Vito Ciaravino, Rachele Cooper, Nathan Copenhaver, Michael Devlin, Vinay D'Souza, Aaron Fishman, Mallory Floyd, Julie Furr, Olivia Gandara, Kathryn Glick, Brian Goldstein, Constantine Hatzis, Åsmund Hellesøy, David Hiett, Danielle Hitchin, Patrick Hunt, Jason Keefer, Franz Kerekes, Jonathan Kettinger, Choong-Yil Kim, David Kirtley, Neal Lepsetz, William Lovis, Enrique Martinez, Gerry Overhiser, Travis Patrick, Erik Preiditsch, Danielle Renton, Kimberly Riggle, Eric Roberts, Craig Rood, Alejandra Salinas, Justin Schnettler, Charles Schwarze, Sarah Shull, Steven Starnes, Mateusz Stec, Michelle Strehle, Uma Subramanian, Kelly Thompson, Jacob Wilford, Joel Yates

‡Department Chairman, Professor, AIAA member

${ }^{\S}$ Professor

Copyright $@ 2001$ by the American Institute of Aeronautics and Astronautics, Inc. All rights reserved.

\section{Introduction}

The Air Force Research Lab (AFRL) sponsored studies to determine the feasibility of a low cost space system capable of high-resolution Earth imaging. Unfortunately, conventional approaches to space system design have proven too costly to meet these needs. To investigate alternative design methods, the Air Force supported the Earth-View Third Millennium (EV-3M) study. The objective of this study was to design an affordable space system presenting a three-fold increase in imaging capabilities at a three-fold decrease in cost. The EV-3M project focuses on innovative design approaches for such a space system, which is to be used for commercial applications. These innovative approaches need not be limited to a single spacecraft or to monolithic telescopes. This paper presents the design of the University of Michigan's EV-3M Earth-Imaging System, developed during the winter 2001 semester as part of the Aero 483/583 Space System Design course.

The Air Force Research Lab issued a Statement of Work $^{1}$ detailing several requirements that our image system had to meet. We analyzed those requirements and, in addition, imposed more stringent goals upon ourselves. Specifically, we aimed at imaging to a resolution of $0.3 \mathrm{~m}$ in the visible band, with an area coverage-rate at this resolution of $400 \mathrm{~km}^{2}$ per day and a revisit time of less than 12 hours. We designed for a signal to noise ratio greater than 40 , and the ability to image through the visible and near IR wavelengths. Moreover, we imposed the goal of a total production cost of less than $\$ 10$ million per satellite. 


$\begin{array}{ll}\text { Nomenclature } \\ c & \text { Speed of light } \\ k & \text { Wave number } \\ t & \text { Time } \\ I & \text { Image surface } \\ O & \text { Observation surface } \\ P & \text { A point } \\ Q & \text { Point source } \\ \alpha & \text { Inclination factor } \\ \lambda & \text { Wavelength of light } \\ \varepsilon & \text { Orbital elevation angle } \\ \omega & \text { Wave frequency } \\ s_{K} & \text { Distance between a point source and the point at } \\ & \text { which it is observed } \\ \hat{n}_{Q} & \text { Unit vector normal to the image surface at } \mathrm{Q} \\ \hat{s}_{Q} & \text { Unit vector pointing from } \mathrm{P} \text { to } \mathrm{Q} \\ i & \text { imaginary number, } \sqrt{-1}\end{array}$

\section{Overall System Description}

We designed our spacecraft around a graphite-epoxy truss two meters long, $0.3 \mathrm{~m}$ wide, and $0.2 \mathrm{~m}$ deep. We placed the majority of the spacecraft components within this structure. The spacecraft will always be pointing toward earth, although not always at Nadir. We designed a 3-axis stabilization system using reaction disks for attitude control, magnetic torquers for momentum dumping, and a flywheel for spin control. The entire spacecraft will be spinning at $1.667 \mathrm{~Hz}$, and the counter-rotating flywheel will control the spin rate. This flywheel will be made to spin at approximately $57,000 \mathrm{~Hz}$ and will keep the spacecraft at zero angular momentum. A computer will be used to control the reaction disks so as to provide the appropriate torques for attitude control despite the high spin rate. A star scanner, IMU, and GPS unit will be used to determine attitude and location. For power generation, we chose a Gallium-Arsenide solar array, and power storage will be provided by Lithium Ion batteries.

We designed a spacecraft featuring a 3-mirror, asymmetric, linear optics array. The optics system uses a Silicon Eye wavefront reconstructor chip ${ }^{4}$. This chip

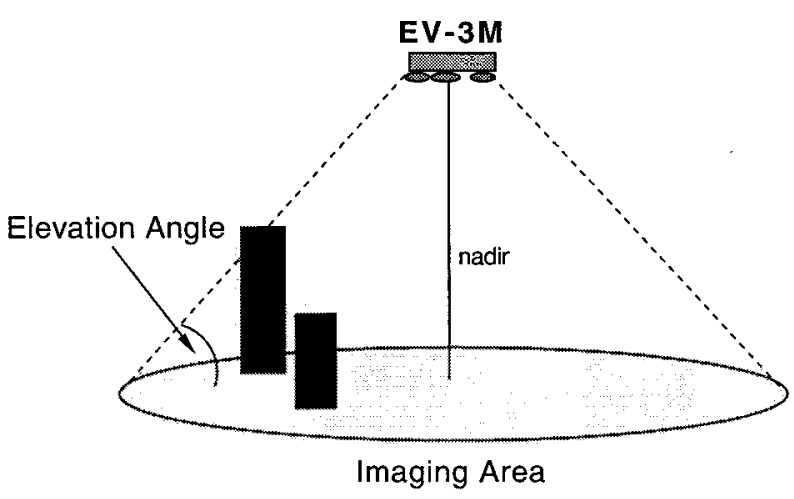

Figure 1. The Elevation Angle, $\varepsilon$

is currently in development at the Air Force Office of Space Research (AFOSR) and promises a substantial increase in capabilities over homothetic recombination and interferometric techniques. In addition to traditional multi-spectral imaging, this chip also determines depth and polarization. This allows us to use the imaging system to create three-dimensional representations of the Earth, as well as to identify polarized light sources. This will enable our spacecraft to differentiate between manmade and natural objects. The optics system is the main focus of this paper and will be addressed in more detail in the following sections.

The satellites will be launched into a $550 \mathrm{~km}$, sunsynchronous orbit ${ }^{12}$. The exact number of satellites launched in our constellation was left undetermined. However, the number of satellites in the constellation is driven by the lowest acceptable elevation angle. As such, wherever possible, we have designed our satellite independent of the final number of satellites chosen. However, for the purpose of this paper, we have proposed a two-satellite baseline configuration that meets all requirements.

The elevation angle is the angle between the lineof-sight to the spacecraft and the horizon plane of the imaging area (see Figure 1). For practical reasons, this elevation angle must be kept smaller than a tolerance, for instance when taking images of large metropolitan areas where the skyscrapers could potentially block out parts of the image.

With a baseline example of two micro-satellites, we recommend launching aboard a Pegasus XL. Some degree of deployment is required in order to fit into the Pegasus fairing. These satellites will be spaced at 
opposite sides of their orbital plane by one satellite performing a small transfer burn. All satellites will use a cold-gas propulsion system using $\mathrm{N}_{2}$ gas. This system will be used for both the deployment maneuver as well as orbital maintenance (required because of atmospheric drag). This configuration will meet all Air Force requirements, as well as all of our additional goals.

The cost per satellite, for materials alone, is estimated to be under $\$ 3$ million and the total mass of each micro-satellite is estimated to be under $50 \mathrm{~kg}$ and will consume less than $130 \mathrm{~W}$ of power.

This satellite configuration allows for continuous earth-coverage, meaning that the entire earth can be imaged every twelve hours. However, this assumes night-time photographs are acceptable. A 24-hour revisit time for daytime photos will be achieved as well.

\section{Mission Design and Sub Systems}

The designed space system consists of a constellation of satellites. The final constellation could consist of any number of satellites. This two-satellite configuration is the minimum required to meet all listed AFRL requirements ${ }^{1}$. These satellites will be spaced evenly in a $550 \mathrm{~km}$, sun-synchronous orbit. Each satellite is identical and carries a 3-mirror, asymmetric, linearly arranged telescope system. The three individual telescopes operate together using a Silicon Eye imaging system. Attitude control is accomplished by use of flywheels and reaction disks. A cold-gas propulsion system is used for orbital maintenance.

\subsection{Optics Payload}

Our optics payload includes three composite mirrors linearly arranged on the spacecraft asymmetrically. The entire system will be spinning at $1.667 \mathrm{~Hz}$. Each composite mirror is $0.288 \mathrm{~m}$ in diameter and has an approximate mass of $0.41 \mathrm{~kg}$. This system is capable of viewing the entire panchromatic spectrum, and can also determine topography and polarization ${ }^{6}$. Our imaging system makes use of a novel imaging technology known as the Silicon Eye, which is based on wave front reconstruction.

\subsection{Structures and Materials}

The majority of spacecraft components are contained in a graphite-epoxy truss, with dimensions $2 \mathrm{~m}$ long, 0.3 $\mathrm{m}$ wide, and $0.2 \mathrm{~m}$ deep. The spherical cold-gas tank, communications antennas, and mirrors are located on the Earth-facing side of the truss, and the solar array is mounted on the top and sides. The entire system has an approximate mass of $35.2 \mathrm{~kg}$.

\subsection{Trajectory Analysis and Propulsion}

We determined that a $550 \mathrm{~km}$, sun-synchronous orbit is optimal for a two-satellite configuration. The actual launch system will be determined after the constellation size is chosen. However, for our 2-satellite baseline example, we recommend the Pegasus XL launch vehicle, which will deliver our two satellites directly to the 550 $\mathrm{km}$ orbit. A maneuver will have to be completed to correctly separate the spacecraft in the orbit. The time required to complete this transfer can vary depending upon requirements, but for our two-satellite example is designed around a 1-month transfer. This is the worst-case scenario, since it requires that one satellite move to the opposite side of the orbit. The satellite will be fully functional during this maneuver, and the only consequence will be non-continuous area coverage until the maneuver is completed. Once in the correctly spaced orbit, coldgas systems will be used for orbital maintenance for the individual satellites.

\subsection{Power and Thermal}

The power system will consist of Ga-As solar arrays and Lithium-Ion batteries. Our design was based on the worst-case, "all systems on" peak power requirement of $123 \mathrm{~W}$. The solar arrays will have a maximum area of $3.7 \mathrm{~m}^{2}$ on the outward side and an area of 3.1 $\mathrm{m}^{2}$ on the earth facing side. The total mass of the solar arrays will be $7.36 \mathrm{~kg}$. The secondary source of power will be Lithium-Ion batteries. These batteries are not yet space qualified but will be for low Earth Orbit (LEO) and geostationary Earth orbit (GEO) by the year 2010 . The total mass of the batteries will be $0.68 \mathrm{~kg}$.

The thermal control system uses both passive and active thermal control. EV-3M systems require a temperature range of $-10^{\circ} \mathrm{C}$ to $25^{\circ} \mathrm{C}$ for operation. The earth facing side of the satellite will be covered with white

3

American Institute of Aeronautics and Astronautics 
enamel and the outward side will be covered with Ga-As solar cells. This provides a temperature range of $-30^{\circ} \mathrm{C}$ to $59^{\circ} \mathrm{C}$. This range was too broad, so we chose to include an active control system as well. The active control system contains conductive switches, radiators, and heaters. The active control system requires $5 \%$ of the total power, which is approximately $7 \mathrm{~W}$. This will keep EV-3M in the specified temperature range needed for operation of all systems.

\subsection{Guidance, Navigation, Command, Control, and Communications}

The on-board sensors and actuators provide active 3axis attitude control within the accuracy requirements of the optical payload. A star scanner, an inertial measurement unit, a GPS receiver, and a magnetometer gather position, acceleration, and rotation information. Reaction wheels, magnetic torque coils, and a momentum wheel provide necessary attitude maintenance and optical payload slewing while maintaining zero total angular momentum. The flywheel is used to control the spacecraft's $1.667 \mathrm{~Hz}$ spin rate. The magnetic torque coils, in turn, are used for momentum dumping, when necessary. We chose the Quadrifilar Helix Antenna as our communications device ${ }^{1}$. This antenna will transmit seven times daily at a data-communications rate of $3 \mathrm{Mbits} / \mathrm{second}$.

\subsection{Test and Quality Control}

We estimate an end-of-life reliability of approximately $95 \%$. A number of components have not yet been selected, and we have not yet added redundancy into the final design. This will be done during the final design process.

We also identified several critical components in the optics system that we could test before construction, and designed a series of five technology demonstration experiments. These tests - four ground based that culminate in a space-based demonstration - will allow us to determine the feasibility and reliability of the specified optics system before the final construction decision has been made. Furthermore, these experiments can be performed in conjunction with several ongoing efforts. Several already funded experiments (by NASA, the NSF, and $\mathrm{AFOSR}^{1}$ ) can operate concurrently to reduce the burden of research and development costs.

\subsection{Systems Integration}

While many of the design decisions are relatively independent of each other, two issues stand out as being functions of parameters from several different subsystems: the orbital altitude and the number of satellites.

2.7.1 Trade Study For a given altitude the geometry of the orbits will limit certain imaging capabilities. Obtaining the resolution and signal to noise ratio (SNR) requirements is possible at any altitude at the cost of increasing the mirror diameter and picture integration time. Also, the ground swath that the spacecraft can image increases with altitude. This is where the unknown elevation angle requirement becomes important. A constellation of two satellites can meet all imaging requirements as listed at $550 \mathrm{~km}$, which is why we analyzed that configuration as our baseline example. However, in order to meet revisit-time requirements, the minimum elevation angle falls to about $38^{\circ}$. A constellation of three satellites will achieve a minimum elevation angle of approximately $52^{\circ}$. Please note that these numbers assume an altitude of $550 \mathrm{~km}$. What this produces is a complex trade diagram that involves several key parameters: altitude, minimum elevation angle, mass, cost, revisit-time, and area- coverage rate. With altitude, constellation size, and elevation angle as our variables, and revisit-time, areacoverage rate, cost, and resolution as our constraints, we can determine what the optimal combination of altitude and number of satellites will minimize cost.

2.7.2 Altitude Determination Without a specified elevation angle $(\varepsilon)$, the trade study discussed above cannot be used and we must choose a more simplified approach to optimizing the altitude. One such approach is to assume that cost is directly related to mass, and thus the orbit that minimizes our mass while meeting all imaging requirements (again, this is ignoring $\varepsilon$, which is taken into account with the number of satellites chosen) will be the cheapest, and thus optimal. This optimal altitude for minimum launch mass is found to be ${ }^{1} 550 \mathrm{~km}$.

2.7.3 Constellation Size The dominant design driver for the number of satellites is the elevation angle. The number of satellites determines the area-coverage rate as well; however, two satellites will be sufficient to 
meet this requirement, as in our baseline configuration. And while the area-coverage rate increases with more satellites, it will do so significantly above our goals.

2.7.4 Constellation Optimization Another option for optimizing the constellation configuration is to add more orbits. Our design has focused primarily upon continuous coverage and a 12 -hour revisit time. This has led to an assumption that all spacecraft will be launched on one launch vehicle, and will then be spaced evenly about the same orbit-plane. We chose this assuming that the elevation angle would be important to the Air Force. However, there are other options.

The first option is to keep the same constellation of two satellites in a sun-synchronous orbit (all within the same plane), and then launch another satellite into an equatorial orbit. The elevation angle is most important near the equator, and thus a satellite in an equatorial orbit would alleviate this problem. We could also launch a couple of satellites in nearly equatorial orbits. This is another matter of optimizing the number of satellites and the elevation angle. It also depends on what areas of the Earth the Air Force feels are most important to image.

Another option is to focus on reducing the revisit time. With a constellation of two satellites a the sunsynchronous, same-plane orbit, we will obtain a 12-hour revisit time. If we instead want a 4-hour revisit time, we could launch three constellations of two satellites each into three separate orbital planes. We would launch them such that one constellation would still always pass overhead at the local-sideral "high-noon". The second constellation would be at inclination $+30^{\circ}$ and the third constellation would be at inclination $-30^{\circ}$. It can be shown that, one of the spacecraft would pass over any spot on earth every four hours ${ }^{1}$.

\section{Optical System}

The optical system consisted of 3 mirrors placed asymmetrically along a linear spinning optical bench, as shown in Figure 2. Furthermore three different optical methods were considered in producing the images: interferometry, homothetic recombination, and wavefront reconstruction. Our goal was to select a system that was low in mass, cost and that could provide a resolution capability three times that of current technology.

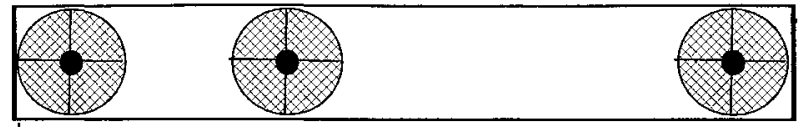

Figure 2. Primary Mirror Layout

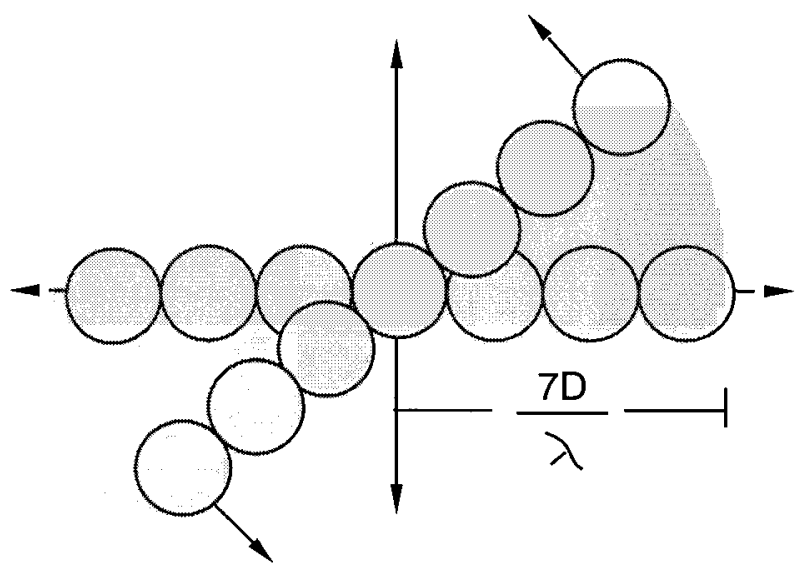

Figure 3. Filled MTF for Synthetic Aperture (wave plane)

The optical bench is spun in order to obtain a complete non-zero Modulation Transfer Function (MTF). In the wave plane this optical bench sweeps out an area equal to that of a single aperture with seven times the diameter of each of the mirrors on the bench, as seen in Figure 3.

\subsection{Wave Optics}

The three optical technologies considered all make use of the wave properties of light. Only one component of the propagating vector wave needs to be considered because all components behave similarly. They propagate according to the wave equation with frequency:

$$
\omega=\frac{2 \pi c}{\lambda}
$$

The value of the field variable at location $x$ is:

$$
\operatorname{Re}\left(U(x, t) e^{-i \omega t}\right)
$$

For a point source, $U(x)=\frac{e^{i k}}{r}$ and the wave number 


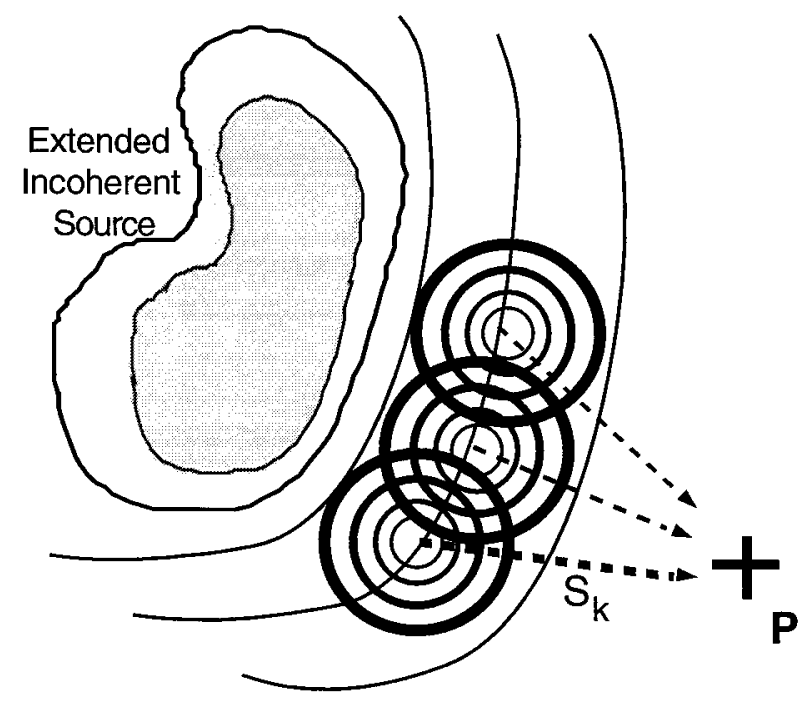

Figure 4. Huygens' Principle

$k=\frac{2 \pi}{\lambda}$. The total field for a point source at position $r$ is then described by:

$$
F=\frac{1}{r} \cos (k r-\omega t)
$$

Huygens' principle asserts that waves emitted from an extended incoherent light source can be thought of as individual point sources each themselves emitting a wave field ${ }^{1}$. That wave field at any point $P$ can be described as a linear combination of contributions from all the point sources. This is graphically shown in Figure 4. This principle is true for optics in general and can be quantified as follows:

$$
U(P)=\frac{i}{\lambda} \sum_{k=1}^{N} \alpha\left(P, Q_{k}\right)\left(\Delta A_{k} U\left(Q_{k}\right)\right)\left(\frac{e^{i k s_{k}}}{s_{k}}\right)
$$

where $\alpha$ is the inclination factor, the term $\Delta A_{k} U\left(Q_{k}\right)$ is the strength and phasing of a point source and $\frac{e^{i k s_{k}}}{s_{k}}$ is the field of a point source at a distance $s_{k}$. Taking the limit as the point sources become infinitely small, then over the wave surface,

$$
U(P)=-\frac{i}{\lambda} \iint_{I} \mathrm{~d} Q \frac{e^{i k s}}{s} \alpha(P, Q) U(Q)
$$

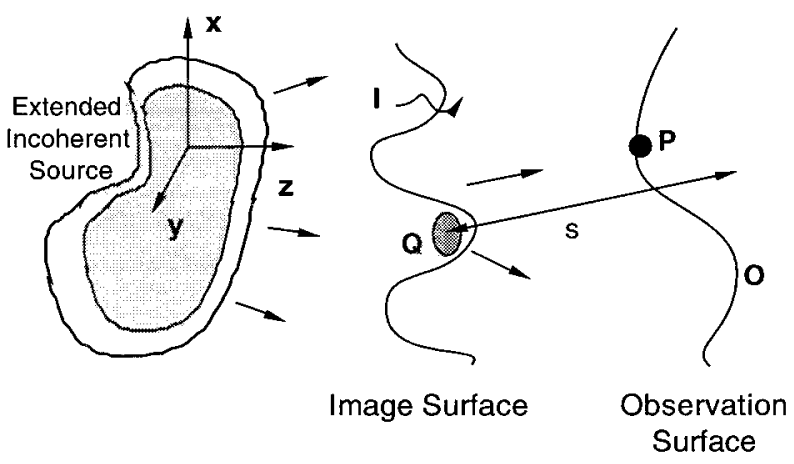

Figure 5. Imaging Situation

\subsection{Imaging Situation}

Figure 5 shows the imaging situation. An extended incoherent light source, $\sigma$, such as the sun, emits the electromagnetic waves that bounce off of the image surface and reach the optical system (in this case low Earth orbit, LEO).

The Huygens-Fresnel Principle ${ }^{1}$ says that if all the waves are nearly perpendicular to the $\mathrm{z}$-axis then the inclination factor, $\alpha(P, Q)$, is given by:

$$
\alpha(P, Q) \approx \hat{n}_{Q} \cdot \hat{s}_{Q}
$$

where $\hat{n}_{Q}$ is the unit vector normal to the image surface at $Q$ and $\hat{s}_{Q}$ is the unit vector pointing from $P$ to $Q$. Substituting Equation 2 into 1 yields:

$$
U(P)=-\frac{i}{\lambda} \iint_{I} \mathrm{~d} Q \frac{e^{i k s}}{s}\left(\hat{n}_{Q} \cdot \hat{s}_{Q}\right) U(Q)
$$

This equation allows us to predict the propagation of light from the imaging surface to any point, including the observation point. However this does not help to recreate the image from the observation point because the wave field at the observation surface would have to be known, which is the ultimate goal of the optical system. Therefore, taking the inverse of Equation 3 allows us to take the observed wave field, $O$, and step it back in time to the desired image surface. This inverse is: 


$$
U(Q) \approx \frac{i}{\lambda} \iint_{O} \mathrm{~d} P \frac{e^{-i k s}}{s}\left(\hat{n}_{P} \cdot \hat{s}_{P}\right) U(P)
$$

Now the observed incoming wave field and polarity can be used to reconstruct the light pattern at the image surface. That is, an image of the Earth in this case.

\section{The Three Optical Technologies}

\subsection{Interferometry}

Interferometry takes light from multiple sources and combines the signals in every combination to construct an image with higher resolution or quality than any of the single sources could produce alone. The interferometric process uses the physical wave properties of light. When two similar waves of light that are in phase are added, they produce a single wave of higher amplitude (brighter light). Conversely if the waves are added and are completely out of phase then the result in a wave with no amplitude or cancel each other out (dark region). This technique requires the use of beam splitters and transfer mirrors behind the main telescopes to combine the light and to focus the combination onto a Charge-Coupled Device (CCD) detector. A schematic for using the layout for our non-symmetric three-aperture system would look like Figure 6 . However this system is very complicated and would require much research and development before it could be used in the application at hand.

\subsection{Homothetic Recombination}

Homothetic Recombination, like interferometry, uses multiple apertures to collect the light of a single "scene" or point source. However, beyond the primary telescopes they look very different. Homothetic Recombination takes the light from each source, reflects the light, and then passes all the beams through another lens (combining telescope) to create one image. The overall idea of this system is that it works just like one large telescope but instead uses multiple apertures to collect the light. Which again saves the mass and expense of using one large mirror. However, the major drawback of this
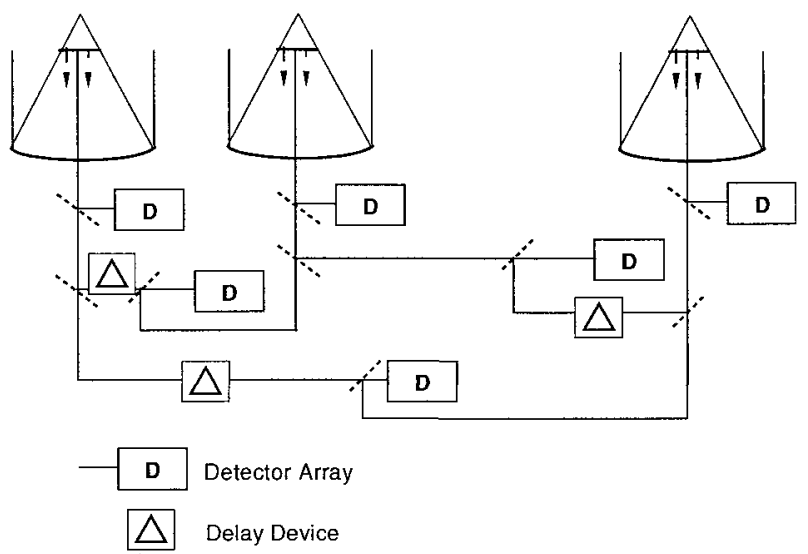

Figure 6. Interferometry Schematic

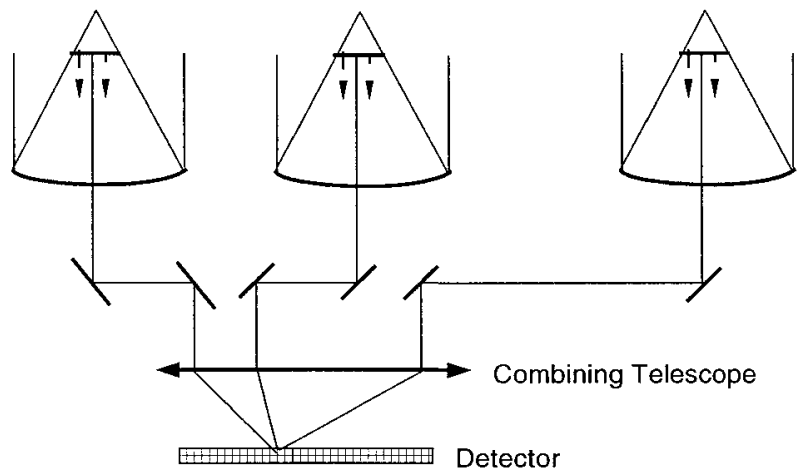

Figure 7. Homothetic Recombination Schematic

technique is that not only does the system need to measure how large of an error there is in the placement of the transfer mirrors, this distance has to be mechanically controlled $^{1}$. Furthermore, this method doesn't support large baselines, so is artificially limited to smaller scale systems ${ }^{11}$. A schematic of homothetic recombination is shown in Figure 7 for our non- symmetric three-aperture system.

\subsection{The Silicon Eye (Wave Front Reconstruction)}

The Silicon Eye technique looks very similar to interferometry when comparing their schematics. Figure 8 is a schematic of the Silicon Eye. However, they are by no means similar in how they process the incoming light to produce an image. This technique is also referred to as wave front reconstruction. Light is collected via numerous apertures and each beam is then added to all of the other light sources exactly like in interferometry. The big difference is that the combined light beams are 


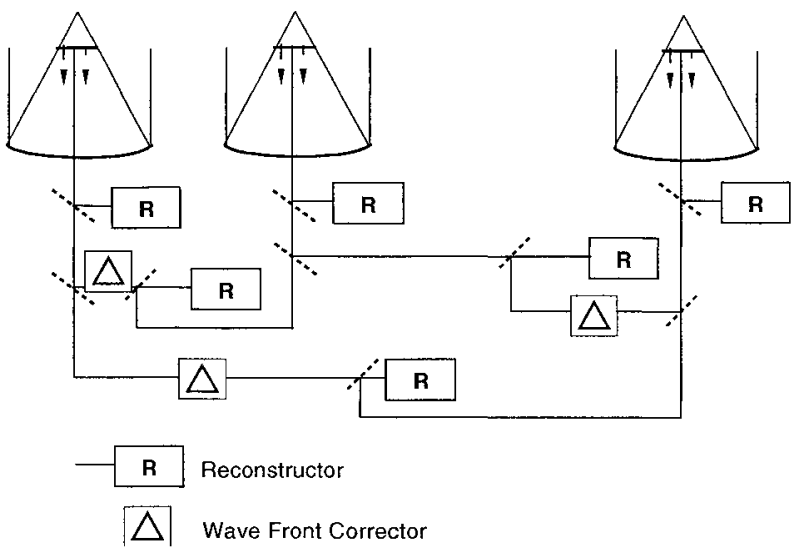

Figure 8. Silicon Eye Schematic

not then detected by a CCD array, but rather a wavefront reconstructor chip. This chip is the "Silicon Eye" and does the reconstructing of the wave using totally analog methods. Reconstructing the wave patterns of the light beams enables us to use Huygens' principle, (4), to find exactly what the light source looked like as it left the objective surface (the surface being imaged). Furthermore, since it is totally analog this process can occur almost instantaneously.

This technique also shifts the paradigm of optical space systems in that larger errors and disturbances can be corrected for, most of the data processing can be done on-line, and also this method can be used on extremely large baselines for extreme precision on very distant objects. It is for all of these reasons that we have chosen this technique for our optical system.

\section{Image Generation}

The light from the three primary telescopes is analogly processed in three different arrays of silicon eyes, or wavefront (WF) reconstructors. These reconstructors themselves consist of two major components a Shack-Hartman wavefront sensor (WFS) and also the wavefront reconstruction chip itself. For each spacecraft there are a given total of $N$ reconstructors. The information from each of the chips is processed in a global WF algorithm. This algorithm produces the sensed wavefront at the observation point. Using (4) this information is used to calculated the wavefront as it was at the desired image plane. This process is called the Image Plane algorithm. From this we take the intensities at each point to construct the image. Also using the

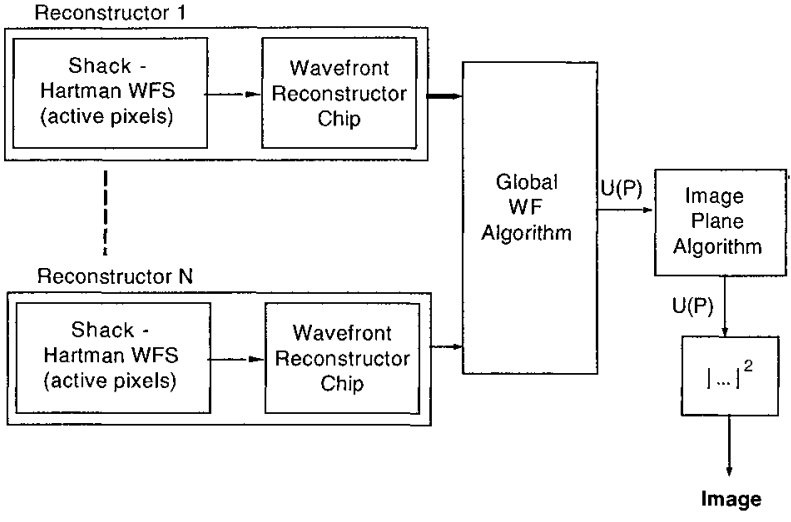

Figure 9. Silicon Eye Image Creation Flow Chart

phase and polarity information detected by the WFS a 3-dimensional hologram can also be produced of the scene. The image creation process is outlined in Figure 9.

\subsection{Metrology}

Real time calibration of the optical system will be necessary to ensure that the images are not blurred and effected minimally by mirror aberrations and optical path length differences. In order to accomplish this a common path approach using phase diversity is used ${ }^{3}$. Sequentially, in time a phase shift device (e.g., liquid crystal variable retarder) introduces shifts at locations where the path length error information is desired. The reconstructor chips collect the phase information for both the shifted and unshifted cases. This information is differenced and phase diversity calculations are done (again using analog processing chips). The result of this is the exact path length correction needed by the imaging algorithm to account for errors in the system.

\section{Conclusions}

The preliminary design presented in this paper has many advantages over current space imaging systems. First it moves away from the conventional monolithic approach, to a low-mass sparse aperture system. In addition, the imaging capabilities are enhanced with the use of the Silicone Eye technology that is being tested and developed at the AFRL, which enable real time image generation with on-line calibration. The low-mass and low-cost of this micro-satelite also enables the use of

8

American Institute of Aeronautics and Astronautics 
constellations at a fraction of the cost of current imaging systems. This reduced cost makes it easier to replace whole satellites that have become damaged or dysfunctional without having to invest major efforts or capital. However, there are many features of this design that need more research and space qualifying tests.

\section{Acknowledgments}

The authors would like to thank Professors James Driscoll, Iain Boyd, Peter Washabaugh, Anthony Wass, Daniel Scheeres, Brian Gilchrist, John Vesechny, and Dr. Robert DiGiovanni for their help. This research effort is supported in part by the Air Force grant F29601-98-D0210 via USRA MBE-00-031/UM/9500-26.

\section{References}

1.- Aero 483/583 Winter 2001 Class, EV-3M Earth Imaging System, Department of Aerospace Engineering Library, The University of Michigan, François-Xavier Bagnoud Building, 1320 Beal Avenue, Ann Arbor, MI 48109-2140.

2.- Belegundu, A. D., Chandrupatla, T. R., Optimization Concepts and Applications in Engineering, Prentice Hall, 1999.

3.- Clark, N., Banish, M., Ranganath, H. S., Smart Optic Systems Using Spatial Light Modulators, IEEE Transactions on Neural Networks, Vol. 10, No. 3, pp. 599-603, May 1999.

4.- Clark, N., Furth, P., Design and Performance Evaluation of a Silicon Eye Using Micro-Mirrors, Air Force Research Labratory.

5.- Clark, N., Furth P., Whitfield, G., Comtois, J., McKecknie, S., Michalicek, A., Silicon adaptive optics systems using micro-mirrors, SPIE conference on Adaptive Optical Systems Technologies, Kona, Hawaii, SPIE vol. 3353, pp. 1146-1153, March 1998.

6.- Clark, N., Prasad, N. S., Dynamic Wavefront Sensor System Using Spatial Light Modulators, SPIE vol. 2566, pp. 54-62. 1995.

7.- Dowling, N.E., Mechanical Behavior of Materials, 2 edition, Prentice-Hall Inc, New Jersey, 1999.

8.- Kopka, H., Daly, Patrick W., A Guide to $L T_{E} X$, Addison-Wesley, third edition 1999.

9.- Lamport, L., IATEX: a Document Preparation System, Reading, MA, Addison-Wesley Pub. Co., 1986.

10.- Reklaitis, G.V., Ravindran, A., Ragsdell, K.M., En- gineering Optimization Methods and Applications, Wiley, 1983.

11.- Traub, W. A.,Combining Beams From Separated Telescopes, Applied Optics, Vol. 25, No. 4, 1986.

12.- Wertz, J. R., Larson, W. J., Space Mission Analysis and Design, Third Edition, Microcosm Press, CA, 1999. 